\title{
Aircraft Ditching Loads Simulation Tool
}

\author{
Antonino Bonanni ${ }^{1, \mathrm{a}}$, Lorenz Vandewaeter ${ }^{1, \mathrm{~b}}$, Caroline Havill ${ }^{1, \mathrm{c}}$, Prin Kanyoo ${ }^{2, \mathrm{a}}$ \\ Dominic Taunton ${ }^{2, b}$, James I.R. Blake ${ }^{2, c}$, Evelyn Cropper $^{1, d}$, Simon Hancock ${ }^{1, e}$ \\ ${ }^{1}$ Stirling Dynamics Ltd, 26 Regent Street BS8 4HG, Bristol, UK \\ ${ }^{2}$ Fluid Structure Interaction Dept., University of Southampton, Southampton, UK \\ 1,aabonanni@stirling-dynamics.com, , 1,blvandewaeter@stirling-dynamics.com, \\ 1,c chavill@stirling-dynamics.com, ${ }^{2, a}$ p.kanyoo@soton.ac.uk, ${ }^{2,0}$ djt2@soton.ac.uk \\ 2,cj.i.r.blake@soton.ac.uk, ${ }^{1, d}$ ecropper@stirling-dynamics.com, ${ }^{1, e}$ shancock@stirling-dynamics.com
}

\section{Keywords: Aircraft Ditching, CFD, Aircraft Loads, Doublet Lattice Method.}

\begin{abstract}
The present work presents a novel methodology developed for calculating the steady loads acting on aircraft structures in the event of ditching in water. It represents the preliminary result of Stirling Dynamics as part of a NATEP research project. The overall objective of the project is to expand the capabilities of the Stirling Dynamics proprietary software SD-GLOAD (originally designed for ground and crash loads dynamic simulations) to aircraft ditching simulations. The methodology presented in this paper employs a Doublet Lattice Method (DLM) to calculate the steady pressure distribution acting on the submerged parts of the ditching aircraft. The proposed methodology is validated against a higher-fidelity CFD multi-phase model for a selected test-case and several ditching conditions.
\end{abstract}

\section{Introduction}

The ability of an aircraft to land safely on water such that the structure stays intact and remains on the surface of the water long enough for the passengers to escape is a mandatory design regulation. This is demanded in regulation, EASA CS 25.801. Although the calculation of ditching loads is part of the aircraft certification process, few validated off-the-shelf commercial tools, which will satisfy the certification authorities, exist to undertake this analysis and, typically, manufacturers have relied on expensive test programmes, or the extrapolation of previous test data to support the new designs. Therefore, the need emerges for more efficient simulation tools that can aid the design engineers to estimate the performances of the airframe during ditching at earlier stages of the aircraft design process. In addition, fluid-structures interaction is likely to play a key role in this analysis. This last aspect is of increasing importance as future aircraft programmes are likely to utilise more flexible airframes. The methods being developed for research and commercial purposes are mostly focused on generating a limited set of high-fidelity CFD data applied to structural models to substantiate the structural integrity. Over the years many authors have improved and tested the CFD models for aircraft ditching analysis based both on classical RANS models [1] and more innovative Smoothed Particle Hydrodynamic SPH models [2][3][4][5][6]. These CFD models are considered more accurate as they are able to retain most of the physics involved in the interaction of multiphase air-water flow (e.g. flow separation, surface tension, cavitation, etc.), but are also computationally intensive. To overcome this lack of suitable tools, Stirling Dynamics have been granted funds from the National Aerospace Technology Development Program (NATEP) to develop an efficient ditching loads simulation tool within their well-established ground and crash loads tool SD-GLOAD. The overall objective of this research project is therefore the development of a methodology for coupling the flexible structure and the hydrodynamic forces in a semi-empirical form. This approach will result in a tool to be used for loads prediction, validation and sensitivity studies, particularly in determining the best angle of approach to maximize passenger survivability. The project has been split in three phases. Phase I focuses on the development of an efficient methodology for steady loads calculation, Phase II 
extends the calculation to take into account the effect of impact loads (due to the vertical component of the aircraft velocity) and Phase III deals with the synthesis of the calculated loads into semi-empirical loads functions that will be integrated within the SD-GLOAD simulation environment for aeroelastic calculations. The present paper focuses on the calculation of the hydrostatic and steady hydrodynamic loads (Phase I). The implementation of the methodology is realized in the Matlab programming environment. The following sections present the methodology based on Doublet Lattice Method (DLM) [7][8] and the validation runs performed against a higher-fidelity CFD model.

\section{Methodology}

Aircraft Geometry The aircraft geometry is represented by a 3D triangulated surface representing the main components of the aircraft (fuselage, wings, HTP and fin). An example of full aircraft geometry is depicted in Fig. 1. The geometry is generated within the Matlab software.

Hydrostatic Loads The hydrostatic loads are calculated by integrating the pressure loads acting on each element of the triangulated surface of the aircraft geometry (Fig. 1) for each given aircraft depth and attitude. The water surface is represented by a plane (and a reference point) and the relative depth of each triangle is then calculated with respect to the plane. Each triangle can then result in fully submerged, partially submerged or out of the water condition. The pressure acting on each triangle is then integrated over the actual submerged portion of its area.

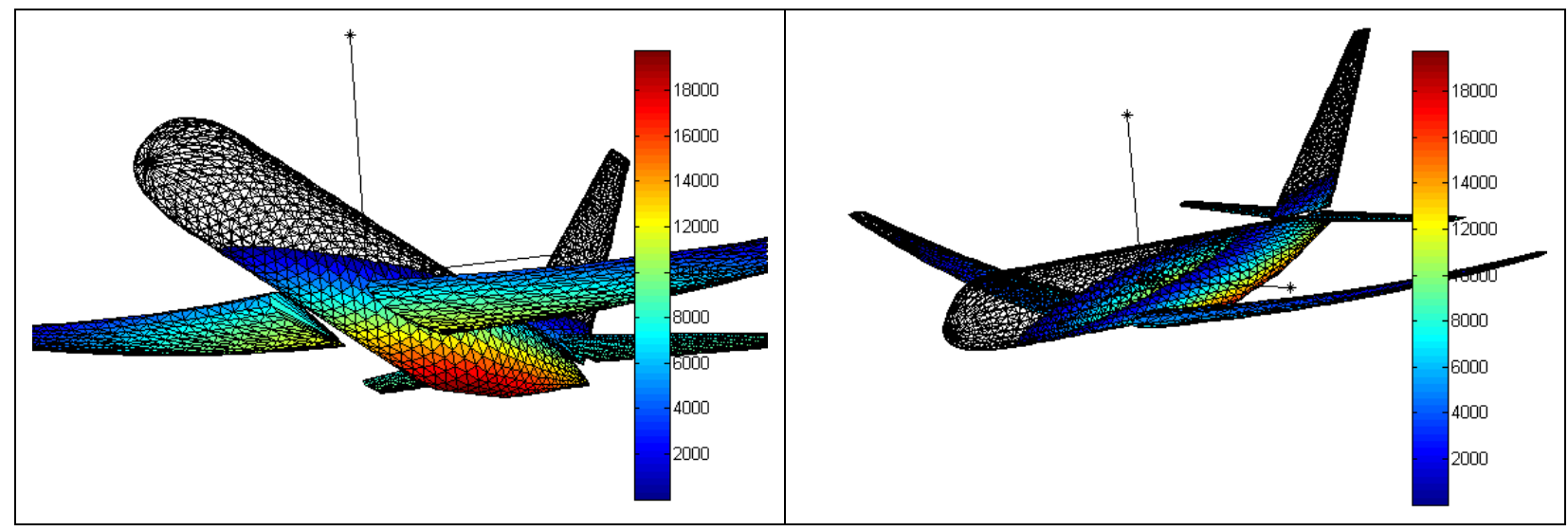

Figure 1. Hydrostatic loads are calculated over the triangulated surface of the 3D geometry.

Hydrodynamic Loads The hydrodynamic loads acting on the airframe during the ditching phase are calculated by solving the inviscid incompressible flow around the airframe by a Doublet Lattice Method DLM technique. This approach differs from previous works (e.g. [7]) as it accounts for an estimation of the water surface deformation during ditching (Fig. 2).

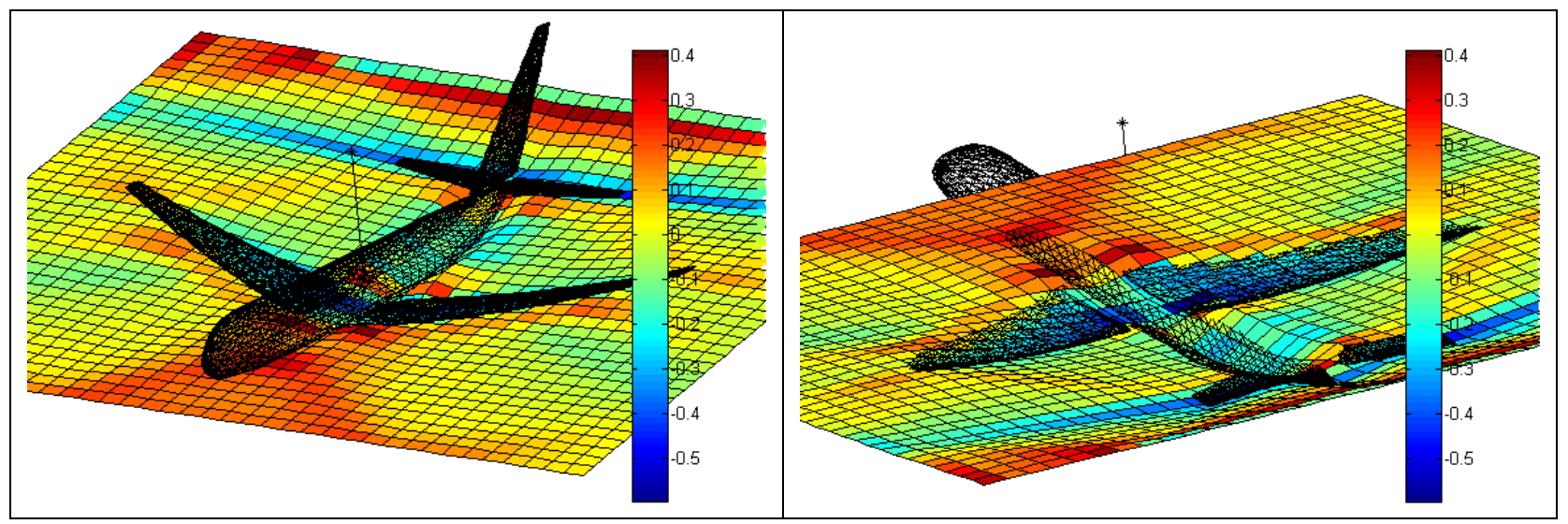

Figure 2. Hydrodynamic loads are derived from the pressure distribution of the DLM surface. 
The doublets are distributed on a grid that approximates the water surface deformed by the aircraft motion. The surface is created by calculating the submerged portion of the aircraft and then interpolating the depths of each submerged triangle by a cubic surface spline defined on a selected portion of the water plane (Fig. 2). The actual DLM problem is then solved on a surface that is composed by the interpolating surface and its mirrored copy with respect to the undisturbed water plane. This last step is needed to enforce symmetry to the potential flow generated by the doublets. Once the flow is made symmetric with respect to the water plane, the induced velocities at each point on the water plane are parallel to the undisturbed water plane itself (reflecting therefore the steady horizontal motion of the aircraft through the water). This approach is deemed more accurate for the pressure distribution on the submerged part of the aircraft (where the interpolating spline well approximates the aircraft geometry) and less in the wake region where no information are available on the actual shape of the water wake.

\section{Model Validation}

CFD Fuselage Simulation The developed model has been validated against a Computational Fluid Dynamic CFD model. The CFD simulations have been performed on a fuselage geometry with the software CD-Adapco StarCCM+. The simulations feature an Eulerian Volume of Fluid VOF model for air and water phases. The runs have been performed for three horizontal steady velocities and no vertical velocity component.

\section{CFD Simulations Parameters}

\begin{tabular}{|l|l|}
\hline Fuselage Length & $23 \mathrm{~m}$ \\
\hline Pitch angle $\theta$ (ref. fuselage center line) & $10^{\circ}$ \\
\hline Reference depth (ref. volumetric CTR) & $0 \mathrm{~m}$ \\
\hline Reference horizontal speed $\mathrm{U}_{0}$ & $5 \mathrm{~m} / \mathrm{s}, 10 \mathrm{~m} / \mathrm{s}, 50 \mathrm{~m} / \mathrm{s}$ \\
\hline CFD Mesh type & Unstructured Cartesian \\
\hline Turbulence Model & URANS $-\mathrm{k}-\varepsilon$ \\
\hline PDE discretization order & Second order in space, first order in time \\
\hline
\end{tabular}

Table 1. Simulation parameters of the reference CFD simulations (CFD RANS VOF model).

Table 1 summarizes the main parameters of the simulations. The pressure coefficient $\mathrm{C}_{\mathrm{p}}$ due to the hydrodynamic effect is defined as follows:

$$
\mathrm{C}_{\mathrm{p}}=\left(\mathrm{p}-\mathrm{p}_{0}\right) /\left(1 / 2 \rho \mathrm{U}_{0}^{2}\right)-(\rho \mathrm{gh}) /\left(1 / 2 \rho \mathrm{U}_{0}^{2}\right)
$$

Where $\mathrm{p}$ is the local pressure, $\mathrm{p}_{0}$ the ambient reference pressure, $\rho$ the density of the water, $\mathrm{h}$ the local depth and $g$ the gravitational acceleration. The contour plot of the pressure coefficient $C_{p}$ for the simulation at $U=10 \mathrm{~m} / \mathrm{s}$ is shown in Fig. 3 .

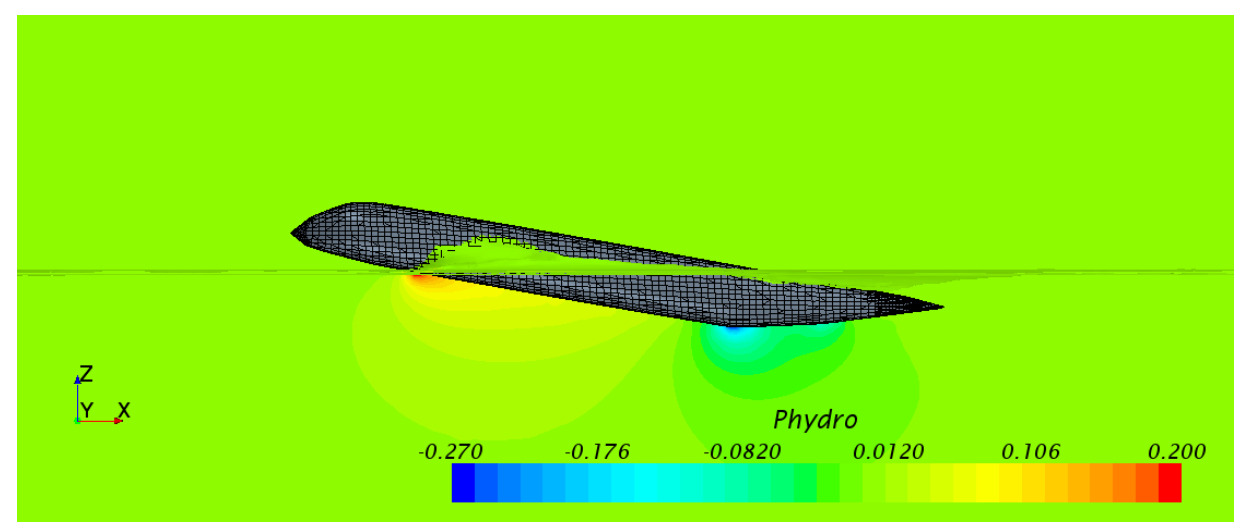

Figure 3. CFD (VOF) model used to simulate airframe loads for steady conditions. 
It should be noted that, if the horizontal velocity is sufficiently high (e.g. for the case $\mathrm{U}_{0}=50 \mathrm{~m} / \mathrm{s}$ ), cavitation effects might need to be taken into account in the CFD model. Nevertheless they have been neglected for the present analysis. From Fig. 3 is possible to observe that the pressure sharply increases at the point where the fuselage first intersects the water plane. In that point the flow is sharply deviated downwards and results in a compression point. The $\mathrm{C}_{\mathrm{p}}$ shows a drop at the point where the tail cone starts. This is an important feature of the ditching fuselage dynamics as it generates a suction effect that can result in a nose-up moment just after the touch down [6].

Loads validation against CFD The test-case reported in Table 1 has then been simulated with the developed Matlab tool and the results of the $C_{p}$ distribution at the bottom line of the fuselage are shown in Fig. 4.

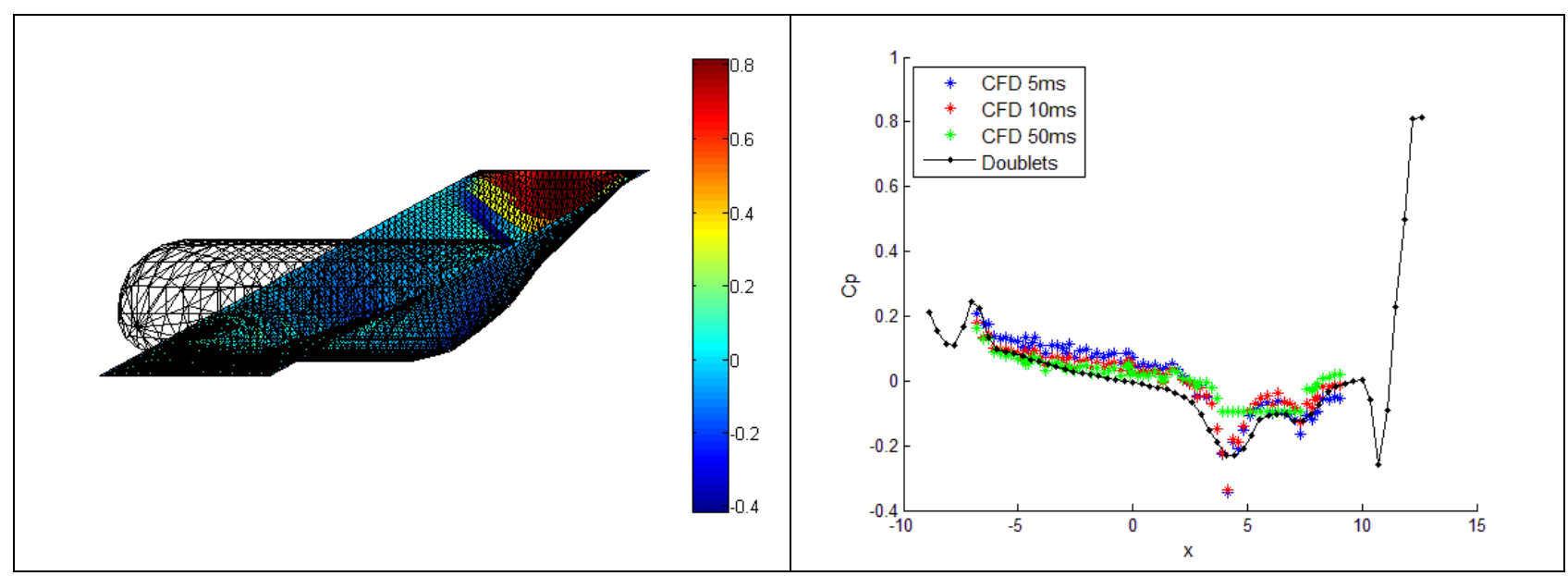

Figure 4. Fuselage test-case: $C_{p}$ distribution (left) and comparison plot against CFD (right).

It is possible to observe that the overall trend of the pressure coefficient is well captured, including the pressure drop at the tail cone point that leads to a suction effect. Nevertheless towards the back of the sampling line (water wake region), the DLM model results in high values of pressure coefficient. This is possibly due to the sharp slope of the interpolating doublet surface at the back of the submerged part (Fig. 4, on the left) and needs to be further investigated. Nevertheless, these high peaks do not affect the loads on the airframe as they happen further downstream of the submerged part of the fuselage.

\section{Conclusions}

A novel technique for aircraft ditching steady loads calculation is presented. The technique is used to calculate the pressure distribution over the deformed water surface by employing a Doublet Lattice Method. It represents the initial step (Phase I) of a NATEP research project that Stirling Dynamics in involved in. The overall objective of the project is the integration of the ditching loads calculation capability in the Stirling Dynamics ground and crash simulation tool SD-GLOAD. The additional steps required will be the calculation of impact loads (Phase II) and the synthesis of the loads into simplified relations for efficient aeroelastic calculations (Phase III). The methodology presented in this work has been validated against a higher fidelity CFD VOF model.

\section{Acknowledgements}

The research presented here represents part of the achievements obtained by Stirling Dynamics within the project funded by the UK National Aerospace Technology Development Program NATEP. The other partners of this project are the University of Southampton and Bombardier Aerospace. 


\section{References}

[1] H. Streckwall, O. Lindenau, L. Bensch. (2007). Aircraft ditching: a free surface / free motion problem. Archives Of Civil And Mechanical Engineering, Vol VII Nb 3.

[2] H.Climent, L.Benitez, F.Rosich, F.Rueda, N.Pentecote. (2006). Aircraft Ditching Numerical Simulation, ICAS 2006.

[3] Pentecote N. Validation of PAM-CRASH code for the simulation of the impact on water. DLR IB 435.2003/3, Jan. 2003.

[4] Rueda F. (IDOM), Peraita C. (IDOM) \& Climent H. CN-235-300M Deepwater - Ditching preliminary water pressure loads. EADS-CASA NT-3-AA0-03006, issue A. Sept. 2003

[5] A R. Ortiz, J.L. Charles, J.F. Sobry (2004). Structural Loading Of A Complete Aircraft Under Realistic Crash Conditions: Generation Of A Load Database For Passenger Safety And Innovative Design. ICAS 2014

[6] Paul H.L.Groenenboom, James Campbell, Luis Benítez Montañés And Martin Siemann (2014). Innovative SPH Methods For Aircraft Ditching. 11th World Congress on Computational Mechanics (WCCM XI), 5th European Conference on Computational Mechanics (ECCM V), 6th European Conference on Computational Fluid Dynamics (ECFD VI).

[7] Farhad Ghaffari (1988). An Analytical Method for Ditching Analysis of an Airborne Vehicle, NASA Report 4120. Langley Research Centre under Contract NAS1-17919.

[8] B.Maskev. (1982). A Computer Program for Calculating the Non-Linear Aerodynamic Characteristics of the Arbitrary Configurations. User Manual NASA Report 8788. 